\title{
On standard forms of 1-dominations between knots with same Gromov volumes
}

\author{
MICHEL BOILEAU, boileau@picard.ups-tlse.fr \\ Laboratoire Émile Picard \\ Université Paul Sabatier, TOULOUSE Cedex 4, France \\ YI NI, yni@math.columbia.edu \\ Department of Mathematics, Columbia University \\ MC 4406, 2990 Broadway, New York, NY 10027 \\ SHICHENG WANG, wangsc@math.pku.edu.cn \\ LMAM, Department of Mathematics, \\ Peking University, Beijing 100871 China
}

\section{Introduction and notations}

All knots are in the 3 -sphere $S^{3}$. For basic terminologies in knot theory and in 3-manifold theory, see [R], [He] and [Ja].

We recall the following relation on the set of knots in $S^{3}$ : let $k_{1}$ and $k_{2}$ be two knots, we say $k_{1} \geq k_{2}$, or equivalently say that $k_{1} 1$-dominates $k_{2}$, if there is a proper degree 1 map $f: E\left(k_{1}\right) \rightarrow E\left(k_{2}\right)$, where $E\left(k_{i}\right)$ is the knot exterior of $k_{i}$. If $k_{1} \geq k_{2}$ but $k_{1} \neq k_{2}$, we often write $k_{1}>k_{2}$, or equivalently say that the 1-domination is non-trivial.

Following the classical results of [Wal] and [GL], it is known that the relation $\geq$ is a partial order on knots in $S^{3}$.

In general, when $k_{1} \geq k_{2}$, the relation of $k_{1}$ and $k_{2}$ is not known, and there is no fine description of the degree 1 map, up to homotopy, realizing the 1domination $k_{1}>k_{2}$. Recall that a simplest and a most common construction of 1 -domination of $k_{1}>k_{2}$ is to choose $k_{1}$ to be a satellization of $k_{2}$ and $f$ 
realizing the 1-domination to be the de-satellization, and on the other hand there are many sophisticated constructions, see [Ka], [Ru], BW1], BW2], [BNW], ORS] and so on.

In this note we show that 1-domination between knots is de-satellization under certain conditions.

Theorem 1.1. Suppose that any companion of $k$ is prime. If $k \geq k^{\prime}$ with the same Gromov volume, then $k^{\prime}$ can be obtained from $k$ by finitely many de-satellizations.

The condition of "same Gromov volume" clearly can not be removed, according to the constructions in the papers mentioned above. We will also give a new construction of 1-domination between knots with same Gromov volume to show that the condition "any companion of $k$ is prime" can not be removed.

The corollary below supports a general opinion that the 1-domination partial order reflects the complexity of knots (see a survey [W]). By a theorem of Schubert [Sc], we have

Corollary 1.2. Suppose that any companion of $k$ is prime. If $k>k^{\prime}$ with the same Gromov volume, then $b(k)>b\left(k^{\prime}\right)$, where $b$ is the bridge number.

The paper is organized as follows. After listing some known useful facts, a general study of maps between Seifert pieces and graph pieces in knot complements is given in $\S 2$, Theorem 1.1 will be proved in $\S 3$, and the new construction of 1-domination will be given in $\S 4$. Below we will fix some notions for the remaining sections.

Notation 1.3. For each solid torus in $S^{3}$, we specify its longitude to be the one which is homologous to zero in the complement. Let $k_{1}$ be a geometrically essential knot [R, p110] in an unknotted solid torus $V \subset S^{3}$ and $k_{2}$ be another knot. Let $h: V \rightarrow N\left(k_{2}\right)$ be a longitude preserving homeomorphism, then the new knot $k=h\left(k_{1}\right)$ is called the satellite knot of $k_{2}$, and $k_{2}$ is a companion of $k$.

The reversing process of satellization, given by pinching $E\left(k_{2}\right)$, the exterior of the companion to a solid torus, produces a proper degree one map $f: E(k) \rightarrow E\left(k_{1}\right)$, which will be called a de-satellization.

Notation 1.4. Let $T\left(p_{1}, q_{1} ; p_{2}, q_{2} ; \ldots ; p_{n}, q_{n}\right)$ be the iterated torus knot, which is the $\left(p_{1}, q_{1}\right)$-cable of the $\left(p_{2}, q_{2}\right)$-cable of $\ldots$ the $\left(p_{n}, q_{n}\right)$-torus knot. 
(When we say " $(p, q)$-cable", $p$ denotes the winding number.) The exterior of the knot is denoted by $E=E\left(p_{1}, q_{1} ; p_{2}, q_{2} ; \ldots ; p_{n}, q_{n}\right)$. Let $C=$ $C\left(p_{1}, q_{1} ; p_{2}, q_{2} ; \ldots ; p_{n}, q_{n}\right)$ denote the "iterated cable space", that is, $E$ with an open neighborhood of the singular fiber corresponding to $\left(p_{n}, q_{n}\right)$ removed. $E$ is a graph manifold, the Seifert pieces are denoted by $C\left(p_{1}, q_{1}\right)$, $\ldots, C\left(p_{n-1}, q_{n-1}\right), E\left(p_{n}, q_{n}\right), \partial E=T_{0}$, the JSJ tori are denoted by $T_{1}, \ldots, T_{n-1}$, where $\partial C\left(p_{i}, q_{i}\right)=T_{i-1} \sqcup T_{i}$. $C$ is also a graph manifold, the Seifert pieces are $C\left(p_{1}, q_{1}\right), \ldots, C\left(p_{n}, q_{n}\right), \partial C=T_{0} \sqcup T_{n}$, the JSJ tori are denoted by $T_{1}, \ldots, T_{n-1}$, where $\partial C\left(p_{i}, q_{i}\right)=T_{i-1} \sqcup T_{i}$. Suppose $\alpha$ is a slope on $T_{n}$, then $C(\alpha)=C\left(p_{1}, q_{1} ; \ldots ; p_{n}, q_{n} ; \alpha\right)$ denotes the manifold obtained by Dehn filling along $\alpha$.

$E$ and $C$ are submanifolds of $S^{3}, T_{i}$ bounds a solid torus $K_{i}$ in $S^{3}$. Suppose $\mu_{i} \subset T_{i}$ is the meridian of $K_{i}$, and $\lambda_{i} \subset T_{i}$ is the longitude.

Notation 1.5. Let $D_{0}$ be a disc and $D_{1}, \ldots, D_{n}$ be sub-discs in the interior of $D_{0}$, and denote $\partial D_{i}$ by $c_{i}$, and the $n$-punctured disc $D_{0} \backslash \cup_{i=1}^{n} D_{i}$ by $P_{n}$. Then $\partial P_{n}=\cup_{i=0}^{n} c_{i}$. Note that $P_{1}$ is an annulus. Once $D_{0}$ is oriented, then $P_{n}$ and all $c_{i}$ are oriented.

Notation 1.6. Let $f: M \rightarrow N$ be a map between orientable compact connected $n$-manifolds. We say that $f$ is proper if $f^{-1}(\partial N)=\partial M$. We say that $f$ is allowable if $f$ is proper and the degree of all possible restrictions $f \mid: F \rightarrow S$ have the same sign, where $F$ is a component of $\partial M$ and $S$ is a component of $\partial N$.

Acknowledgements. This research was conducted during the periods the second author was a graduate student at Princeton University and was employed by the Clay Mathematics Institute as a Liftoff Fellow. The second author was partially supported by a Graduate School Centennial Fellowship at Princeton University. The third author is partially supported by grant No.10631060 of the National Natural Science Foundation of China.

\section{Proper maps between Seifert pieces and graph pieces in knot complements}

The following four known facts, see [Go], Ja], Ro] and [So] respectively, will be repeatly used in this paper. 
Lemma 2.1. [Go] In $C\left(p_{i}, q_{i}\right)$, we have the following relations in homology:

$$
p_{i}\left[\mu_{i-1}\right]=\left[\mu_{i}\right], \quad\left[\lambda_{i-1}\right]=p_{i}\left[\lambda_{i}\right] .
$$

Moreover the regular Seifert fiber of $C\left(p_{i}, q_{i}\right)$ is homologous to $p_{i} q_{i}\left[\mu_{i-1}\right]+$ $\left[\lambda_{i-1}\right]$ on $T_{i-1}$, and homologous to $q_{i}\left[\mu_{i}\right]+p_{i}\left[\lambda_{i}\right]$ on $T_{i}$.

Lemma 2.2. [Ja Let $P$ be a Seifert piece of the JSJ decomposition of $E(k)$. Then $P$ is either $E(p, q)$, or $C(p, q)$, or $P_{m} \times S^{1}$. Moreover $P_{m} \times S^{1}, m>1$ appears if and only some companion of $k$ is not prime.

Lemma 2.3. [Ro] Let $f: M \rightarrow N$ be an allowable degree 1 map between aphereical Seifert manifolds. Then $f$ is homotopic to a fiber preserving pinch.

Lemma 2.4. [So] If $f: M \rightarrow N$ is a proper map of degree d between Haken manifolds such that $\|M\|=d\|N\|$, then $f$ can be homotoped to send $H(M)$ to $H(N)$ by a covering, where $\|*\|$ is the Gromov norm and $H(*)$ is the hyperbolic part under the JSJ decomposition.

Below we give some general study of maps between Seifert pieces and graph pieces in knot complements.

Lemma 2.5. Any proper degree 1 map $f: E(p, q) \rightarrow E\left(p^{\prime}, q^{\prime}\right)$ between torus knot complements is homotopic to a homeomorphism.

Proof. The lemma is known since that all torus knots are minimal (see [BW1]). It is also a direct corollary of [Ro]: Since each manifold involved has only one boundary component, $f$ is an allowable degree 1 map. Since each Seifert manifold involved has a unique Seifert fibration, then by [Ro], $f$ is homotopic to a fiber preserving pinch. Since any non-trivial pinch will decrease either the genus of the orbifold, or the number of singular fibers, and since both the genus of the orbifold and the number of singular fibers of $E(p, q)$ and $E\left(p^{\prime}, q^{\prime}\right)$ are the same, the pinch must be trivial, therefore the lemma is verified.

Lemma 2.6. Suppose $M$ is a Seifert manifold with a $\pi_{1}$-injective boundary component $T$ and $f: C\left(p_{1}, q_{1}\right) \rightarrow M$ is a proper map such that $f \mid: T_{0} \rightarrow T$ is a homeomorphism. Let $t_{1} \in \pi_{1}\left(C\left(p_{1}, q_{1}\right)\right)$ and $t \in \pi_{1}(M)$ represent regular fibers of the corresponding Seifert manifolds. Then the following statements hold.

(1) $f_{*}\left(\pi_{1}\left(C\left(p_{1}, q_{1}\right)\right)\right)$ is not an abelian group;

(2) $f_{*}\left(t_{1}\right)=t^{ \pm 1}$ if $M$ has a unique Seifert fibration. 
Proof. Pick a base point of $C\left(p_{1}, q_{1}\right)$ in $T_{0}$, and a base point of $M$ in $T$. Then $\pi_{1}\left(T_{0}\right)$ is naturally a subgroup of $\pi_{1}\left(C\left(p_{1}, q_{1}\right)\right)$, and $\pi_{1}(T)$ is naturally a subgroup of $\pi_{1}(M)$.

Assume $f_{*}\left(\pi_{1}\left(C\left(p_{1}, q_{1}\right)\right)\right)$ is an abelian group. Since $f \mid T_{0}$ is a homeomorphism, and $\pi_{1}(T)$ is a maximal abelian subgroup of $\pi_{1}(M), f_{*}\left(\pi_{1}\left(C\left(p_{1}, q_{1}\right)\right)\right)$ must be $\pi_{1}(T)$. Moreover,

$$
f_{*}: \pi_{1}\left(C\left(p_{1}, q_{1}\right)\right) \rightarrow \pi_{1}(T)
$$

factors through $\mathrm{H}_{1}\left(C\left(p_{1}, q_{1}\right)\right)$. $\lambda_{0}, \lambda_{1}$ represent elements in $\pi_{1}\left(C\left(p_{1}, q_{1}\right)\right), \lambda_{0}$ is the $p_{1}$-multiple of $\lambda_{1}$ in $\mathrm{H}_{1}\left(C\left(p_{1}, q_{1}\right)\right)$, but $f_{*}\left(\lambda_{0}\right)$ is a primitive element in $\pi_{1}(T)$, we get a contradicition.

Since $t_{1}$ commutes with $\pi_{1}\left(C\left(p_{1}, q_{1}\right)\right)$, and $f_{*}\left(\pi_{1}\left(C\left(p_{1}, q_{1}\right)\right)\right)$ is non-abelian, $f_{*}\left(t_{1}\right)$ must be a power of $t$. Since $f: T_{0} \rightarrow T$ is a homeomorphism, $f_{*}\left(t_{1}\right)=$ $t^{ \pm 1}$.

Lemma 2.7. Let

$$
f: C(\alpha)=C\left(p_{1}, q_{1} ; \ldots ; p_{n}, q_{n} ; \alpha\right) \rightarrow E(p, q)
$$

be a proper map, and the restriction of $f$ to $T_{0}$ is a homeomorphism. Then the restriction of $f$ to $T_{1}$ is not $\pi_{1}$-injective.

Proof. Pick a basepoint $b$ of $C(\alpha), b \in T_{0}$, choose a simple curve $\gamma$ connecting $b$ to $T_{n-1}$, such that $\gamma \cap T_{i}$ consists of a single point. Let $\gamma \cap T_{i}$ be the base point in $T_{i}$ and $E\left(p_{i+1}, q_{i+1}\right)$. Using a path on $\gamma$, we can view $\pi_{1}\left(T_{i}\right)$ and $\pi_{1}\left(E\left(p_{i+1}, q_{i+1}\right)\right)$ as subgroups of $\pi_{1}(C(\alpha))$. Let $f_{*}: \pi_{1}(C(\alpha)) \rightarrow \pi_{1}(E(p, q))$ be the induced map on $\pi_{1}$. Let $T_{0}^{\prime}=\partial E(p, q)$.

Let $t_{i} \subset \pi_{1}\left(C\left(p_{i}, q_{i}\right)\right)$ and $t \subset \pi_{1}(E(p, q))$ represent the regular Seifert fibers in the corresponding Seifert manifolds. By Lemma 2.6, we can assume $f_{*}\left(t_{1}\right)=t$.

If $n=1$, then the conclusion trivially holds (since $\alpha$ is in the kernal), so we assume $n>1$. The element $t_{1}$ is contained in $\pi_{1}\left(T_{1}\right)$. In fact, $t_{1}$ is homologous to $q_{1}\left[\mu_{1}\right]+p_{1}\left[\lambda_{1}\right]$ in $T_{1}$. Let $x$ denote $f_{*}\left(\mu_{1}\right)$. Assume the restriction of $f$ on $T_{1}$ is $\pi_{1}$-injective, then $x, t$ generate a $\mathbb{Z} \oplus \mathbb{Z}$-subgroup of $\pi_{1}(E(p, q))$.

The fiber $t_{2}$ is homologous to $p_{2} q_{2}\left[\mu_{1}\right]+\left[\lambda_{1}\right]$ on $T_{1}$, hence not a power of $t_{1}$ in $\pi_{1}\left(T_{1}\right)$. So $f_{*}\left(t_{2}\right)$ is not a power of $t$. But $t_{2}$ commutes with $\pi_{1}\left(C\left(p_{2}, q_{2}\right)\right)$, so $f_{*}\left(\pi_{1}\left(C\left(p_{2}, q_{2}\right)\right)\right)$ is an abelian group. Hence

$$
f_{*}: \pi_{1}\left(C\left(p_{2}, q_{2}\right)\right) \rightarrow \pi_{1}(E(p, q))
$$


factors through $\mathrm{H}_{1}\left(C\left(p_{2}, q_{2}\right)\right)$.

In $C\left(p_{2}, q_{2}\right), p_{2}\left(q_{1}\left[\mu_{1}\right]+p_{1}\left[\lambda_{1}\right]\right)$ is homologous to $q_{1}\left[\mu_{2}\right]+p_{1} p_{2}^{2}\left[\lambda_{2}\right]$, hence the corresponding element in $\pi_{1}\left(T_{2}\right)$ is mapped by $f_{*}$ to $t^{p_{2}}$. By the same reason, $f_{*}\left(\mu_{2}\right)=x^{p_{2}}$. So $f \mid T_{2}$ is $\pi_{1}$-injective. Moreover, $t_{3}$ is homologous to $p_{3} q_{3}\left[\mu_{2}\right]+\left[\lambda_{2}\right]$ in $T_{2}$, it is linearly independent with $q_{1}\left[\mu_{2}\right]+p_{1} p_{2}^{2}\left[\lambda_{2}\right]$, since $\operatorname{gcd}\left(p_{1}, q_{1}\right)=1$. Hence $f_{*}\left(t_{3}\right)$ is not a power of $t$. But $t_{3}$ commutes with $\pi_{1}\left(C\left(p_{3}, q_{3}\right)\right)$, so $f_{*}\left(\pi_{1}\left(C\left(p_{3}, q_{3}\right)\right)\right)$ is an abelian group.

Argue as before, we find that $f_{*}\left(\mu_{3}\right)=x^{p_{2} p_{3}}$, and the loop corresponding to $q_{1}\left[\mu_{3}\right]+p_{1} p_{2}^{2} p_{3}^{2}\left[\lambda_{3}\right]$ on $T_{3}$ is mapped to $t^{p_{2} p_{3}}$ by $f_{*}$. Hence $f \mid T_{3}$ is $\pi_{1}$-injective, and $f_{*}\left(t_{4}\right)$ is not a power of $t$.

Go on with such argument, we finally show that $f \mid T_{n-1}$ is $\pi_{1}$-injective, and $f_{*}\left(t_{n}\right)$ is not a power of $t$, where $t_{n}$ represents the regular fiber of $C\left(p_{n}, q_{n}\right)$. Thus $f_{*}\left(\pi_{1}\left(C\left(p_{n}, q_{n}\right)\right)\right)$ is an abelian group, and therefore the group $f_{*}\left(\pi_{1}\left(C\left(p_{n}, q_{n} ; \alpha\right)\right)\right)$ is also abelian. Then $f_{*} \mid \pi_{1}\left(C\left(p_{n}, q_{n} ; \alpha\right)\right)$ factors through $\mathrm{H}_{1}\left(C\left(p_{n}, q_{n}, \alpha\right)\right) \cong \mathbb{Z} \oplus \mathbb{Z}_{b}$ for some positive integer $b$, which contradicts to the fact that $f \mid T_{n-1}$ is $\pi_{1}$-injective.

Lemma 2.8. Suppose $\partial C(p, q)=T_{0}^{\prime} \sqcup T_{1}^{\prime}$, where $T_{0}^{\prime}$ bounds a neighborhood of the torus knot $T(p, q)$, and

$$
f: C\left(p_{1}, q_{1} ; \ldots ; p_{n}, q_{n}\right) \rightarrow C(p, q)
$$

is a proper map.

(1) If $n>1$, then $f$ cannot map $T_{0}$ homeomorphically to $T_{0}^{\prime}$.

(2) If $n=1$, and $f$ maps $T_{0}$ homeomorphically to $T_{0}^{\prime}$, then $f$ is homotopic to a homeomorphism.

Proof. Assume $f$ maps $T_{0}$ homeomorphically to $T_{0}^{\prime}$. We claim that $f\left(T_{n}\right)=$ $T_{1}^{\prime}$. Otherwise $f\left(T_{n}\right)=T_{0}^{\prime}$. Let $f_{\#}$ be the induced map on homology. $f_{\#}\left(\left[\lambda_{n}\right]\right)$ is an integral linear combination of $f_{\#}\left(\left[\mu_{0}\right]\right)$ and $f_{\#}\left(\left[\lambda_{0}\right]\right)$, but $\left[\lambda_{n}\right]$ is equal to $\frac{1}{P}\left[\lambda_{0}\right]$, where $P=p_{1} p_{2} \ldots p_{n}$. We get a contradiction.

Now $f\left(T_{n}\right)=T_{1}^{\prime}$. Since $f \mid T_{0}$ is a homeomorphism, $\operatorname{deg} f=\operatorname{deg} f \mid T_{n}=1$. We can homotope $f$, so that $f \mid T_{n}$ is a homeomorphism. Moreover,

$$
f_{\#}: \mathrm{H}_{1}(C) \rightarrow \mathrm{H}_{1}(C(p, q))
$$

is an isomorphism.

By Lemma 2.6, we can assume $f_{*}\left(t_{1}\right)=f_{*}\left(t_{n}\right)=t$. In $\mathrm{H}_{1}(C)$, we have

$$
\left[t_{1}\right]=p_{1} q_{1}\left[\mu_{0}\right]+\left[\lambda_{0}\right]=p_{1} q_{1}\left[\mu_{0}\right]+P\left[\lambda_{n}\right]
$$




$$
\left[t_{n}\right]=q_{n}\left[\mu_{n}\right]+p_{n}\left[\lambda_{n}\right]=q_{n} P\left[\mu_{0}\right]+p_{n}\left[\lambda_{n}\right] .
$$

Since $f_{\#}$ is an isomorphism and $\left[\mu_{0}\right],\left[\lambda_{n}\right]$ generate $\mathrm{H}_{1}(C)$, we must have

$$
p_{1} q_{1}=q_{n} P, \quad P=p_{n}
$$

If $n>1$, it is impossible since $p_{1}>1$.

If $n=1$, then we have a proper allowable degree map $f: C\left(p_{1}, q_{1}\right) \rightarrow$ $C(p, q)$. Applying Rong's result as in the proof of Lemma 2.5, one shows that $f$ is homotopic to a homeomorphism.

Lemma 2.9. Let $M$ be either $E\left(p_{1}, q_{1} ; \ldots ; p_{n}, q_{n}\right)$ or $C\left(p_{1}, q_{1} ; \ldots ; p_{n}, q_{n}\right)$, let $P_{m}$ denote the $m$-punctured disk, where $m>0$. Then there is no proper map $f: M \rightarrow P_{m} \times S^{1}$ such that $f$ restricts to a component of $\partial M$ is a homeomorphism.

Proof. Assume $f$ maps $T_{0}$ homeomorphically to $T_{0}^{\prime}$, a component of $\partial P_{m} \times S^{1}$.

If $M$ is a knot space, then $\left[T_{0}^{\prime}\right]=f_{\#}\left(\left[T_{0}\right]\right)$ is null homologous in $P_{m} \times S^{1}$, which implies $m=0$, a contradiction.

Now suppose that $M$ is an iterated cable space with boundary $T_{0}$ and $T_{n}$. Since $\left[\lambda_{0}\right]=p\left[\lambda_{n}\right]$ in $\mathrm{H}_{1}(M ; \mathbb{Z})$, where $p=p_{1} \ldots p_{n}>1$, we have $f_{\#}\left(\left[\lambda_{0}\right]\right)=$ $p f_{\#}\left(\left[\lambda_{n}\right]\right)$ in $\mathrm{H}_{1}\left(P_{m} \times S^{1} ; \mathbb{Z}\right)=\mathbb{Z}^{m+1}$. There are two subcases:

(a) $f_{\#}\left(\left[T_{n}\right]\right)=k\left[T_{0}^{\prime}\right], k \in \mathbb{Z}$;

(b) $f_{\#}\left(\left[T_{n}\right]\right)=k\left[T_{1}^{\prime}\right], k \in \mathbb{Z}, T_{1}^{\prime} \neq T_{0}^{\prime}, T_{1}^{\prime}$ is a component of $\partial P_{m} \times S^{1}$.

In the subcase $(\mathrm{a})$, since $\left[T_{0}\right]+\left[T_{n}\right]=0$, we have $(k+1)\left[T_{0}^{\prime}\right]=0$, which implies that $k=-1$. Now both $f_{\#}\left(\left[\lambda_{0}\right]\right)$ and $f_{\#}\left(\left[\lambda_{n}\right]\right)$ are homologous to closed curves on $T_{0}^{\prime}$, and in particular $f_{\#}\left(\left[\lambda_{0}\right]\right)$ is a primitive element in $\mathrm{H}_{1}\left(T_{0}^{\prime} ; \mathbb{Z}\right)=\mathbb{Z}^{2}$. Note that $f_{\#}\left(\left[\lambda_{0}\right]\right)=p f_{\#}\left(\left[\lambda_{n}\right]\right)$ in $\mathrm{H}_{1}\left(P_{m} \times S^{1} ; \mathbb{Z}\right)$, and the homomorphism $\mathrm{H}_{1}\left(T_{0}^{\prime} ; \mathbb{Z}\right) \rightarrow \mathrm{H}_{1}\left(P_{m} \times S^{1} ; \mathbb{Z}\right)$ induced by the inclusion is injective, so $f_{\#}\left(\left[\lambda_{0}\right]\right)=p f_{\#}\left(\left[\lambda_{n}\right]\right)$ in $\mathrm{H}_{1}\left(T_{0}^{\prime} ; \mathbb{Z}\right)$, which is impossible since $f_{\#}\left(\left[\lambda_{0}\right]\right)$ is primitive.

In the subcase $(\mathrm{b})$, since $\left[T_{0}\right]+\left[T_{n}\right]=0$, we have $\left[T_{0}^{\prime}\right]+\left[T_{1}^{\prime}\right]=0$, which is impossible if $m>1$. If $m=1$, then $P_{1} \times S^{1}=T_{0}^{\prime} \times[0,1]$, and the homomorphism $\mathrm{H}_{1}\left(T_{0}^{\prime} ; \mathbb{Z}\right) \rightarrow \mathrm{H}_{1}\left(T_{0}^{\prime} \times[0,1] ; \mathbb{Z}\right)$ induced by the inclusion is an isomorphism, and again $f_{\#}\left(\left[\lambda_{0}\right]\right)=p f_{\#}\left(\left[\lambda_{n}\right]\right)$ in $\mathrm{H}_{1}\left(P_{1} \times S^{1} ; \mathbb{Z}\right)$, which is impossible.

In either case we reach a contradiction. 


\section{Proof of Theorem 1.1}

The dual graph $\Gamma(k)$ to the JSJ-decomposition of $E(k)$ is a rooted tree, where the root is corresponding to the unique vertex manifold containing $\partial E(k)$. Let $\Gamma_{0}(k) \subset \Gamma(k)$ be the maximal connected subtree which contains the root such that the restriction of $f$, up to homotopy, to the connected submanifold $M\left(\Gamma_{0}\right)$ associated to $\Gamma_{0}$ is a homeomorphism to its image, and moreover the restriction of $f$ to each leaf torus of $\Gamma_{0}$ is $\pi_{1}$-injective.

Since $k$ and $k^{\prime}$ have the same Gromov volume, by [So], $f$ can be homotoped so that $f$ maps the hyperbolic pieces of $E(k)$ homeomorphically to the hyperbolic pieces of $E\left(k^{\prime}\right)$.

If $f: E(k) \rightarrow E\left(k^{\prime}\right)$ is homotopic to a homeomorphism, then Theorem 1.1 is automatically true. So below we assume that $f$ is not homotopic to a homeomorphism. Then $M\left(\Gamma_{0}\right) \neq E(k)$.

Let $T_{0}$ be the torus corresponding to a leaf of $\Gamma_{0}$, and $X_{0}\left(\not \subset M\left(\Gamma_{0}\right)\right)$ be the JSJ piece adjacent to $T_{0}$. Then $X_{0}$ must be a Seifert piece. Since $f \mid T_{0}$ is $\pi_{1}-$ injective, $f \mid X_{0}$ is non-degenerate, and it follows that we can push $f\left(X_{0}\right)$ into a Seifert piece $X_{0}^{\prime}$ of the JSJ decomposition of $E\left(k^{\prime}\right)$. Let $T_{0}^{\prime}=f\left(T_{0}\right) \subset \partial X_{0}^{\prime}$, then $f \mid: T_{0} \rightarrow T_{0}^{\prime}$ is a homeomorphism. By the definition of $\Gamma_{0}(k)$, we have a JSJ piece $X \neq X_{0}$ of $E(k)$ adjacent to $T_{0}$ such that $f \mid: X \rightarrow X^{\prime}$ is a homeomorphism, where $X^{\prime}$ is a JSJ piece of $E\left(k^{\prime}\right)$ adjacent to $T_{0}^{\prime}$.

Let $U$ be the maximal connected graph submanifold of $E(k)$ such that $X_{0} \subset U$ and $T_{0} \subset \partial U$. Since we assume that any companion of $k$ is prime, then $U$ is in the form of either $E\left(p_{1}, q_{1} ; \ldots ; p_{n}, q_{n}\right)$ or $C\left(p_{1}, q_{1} ; \ldots ; p_{n}, q_{n}\right)$.

Lemma 3.1. $U \neq E\left(p_{1}, q_{1}\right)$, hence $T_{1} \neq \emptyset$.

Proof. Otherwise we have $U=X_{0}=E\left(p_{1}, q_{1}\right)$ and then $f\left(T_{0}\right)=T_{0}^{\prime}$ is homologous to zero in $X_{0}^{\prime}$, which implies $\partial X_{0}^{\prime}=T_{0}^{\prime}$ and therefore $X_{0}^{\prime}=$ $E\left(p^{\prime}, q^{\prime}\right)$. Then we have map $f \mid: E(p, q) \rightarrow E\left(p^{\prime}, q^{\prime}\right)$ which is degree 1 on the boundary, and therefore degree 1 itself. By Lemma 2.5, $f \mid$ is homotopic to a homeomorphism, and therefore contradicts to the maximality of $\Gamma_{0}$.

Below we name JSJ-tori in $U$ after $T_{1}$ as $T_{2}, \ldots, T_{n}$ in order.

Lemma 3.2. $f \mid T_{i}$ is not $\pi_{1}$-injective for some $i$.

Proof. Otherwise the restriction of $f$ to any Seifert piece in $U$ is non-degenerate. By homotoping $f$, we can assume $f^{-1}\left(X_{0}^{\prime}\right)$ is the union of some Seifert pieces in $E(k)$. 
Let $G$ be a component of $f^{-1}\left(X_{0}^{\prime}\right)$ containing $X_{0}$. The $G$ is either $E\left(p_{1}, q_{1} ; \ldots ; p_{l}, q_{l}\right)$ or $C\left(p_{1}, q_{1} ; \ldots ; p_{l}, q_{l}\right)$.

Claim 1. $X_{0}^{\prime}=E\left(p^{\prime}, q^{\prime}\right)$, and $X^{\prime} \neq X_{0}^{\prime}$.

Proof. By Lemma 2.9, $X_{0}^{\prime}$ is not $P_{m} \times S^{1}, m \geq 1$. Hence either $X_{0}^{\prime}=C\left(p^{\prime}, q^{\prime}\right)$ or $X_{0}^{\prime}=E\left(p^{\prime}, q^{\prime}\right)$.

Suppose first $X_{0}^{\prime}=C\left(p^{\prime}, q^{\prime}\right)$. By simple homological reason $G$ cannot be $E\left(p_{1}, q_{1} ; \ldots ; p_{l}, q_{l}\right)$. By Lemma 2.8 , $G$ cannot be $C\left(p_{1}, q_{1} ; \ldots ; p_{l}, q_{l}\right), l>1$; moreover if $C=C\left(p_{1}, q_{1}\right)$, then $f \mid: C\left(p_{1}, q_{1}\right) \rightarrow C\left(p^{\prime}, q^{\prime}\right)$ is homotopic to a homeomorphism, which contradicts to the maximality of $\Gamma_{0}$.

Hence $X_{0}^{\prime}=E\left(p^{\prime}, q^{\prime}\right)$. Since $X^{\prime}$, which is homeomorphic to $X$, has at least two boundary components, we have $X_{0}^{\prime} \neq X^{\prime}$.

Claim 2. $f^{-1}\left(X_{0}^{\prime}\right) \cap U=U$.

Proof. Let $S^{\prime}$ be a Seifert surface of $E\left(p^{\prime}, q^{\prime}\right)$. Since $f \mid: T_{0} \rightarrow T_{0}^{\prime}$ is a homeomorphism, up to a homotopy relative to $T_{0}$, we may assume that $f^{-1}\left(S^{\prime}\right)$ is incompressible, and moreover there is only one component of $f^{-1}\left(S^{\prime}\right)$, denoted by $S$, with $\partial S$ a circle $c$. Since $f(X)=X^{\prime}, X^{\prime} \neq X_{0}^{\prime}$, it follows $f^{-1}\left(S^{\prime}\right) \cap \operatorname{int} X=\emptyset$. Since $T_{0}$ is separating and $S$ is connected, we must have $S \subset E\left(k_{0}\right)$, hence $c=\lambda_{0}$, where $E\left(k_{0}\right)$ is a component separated by $T_{0}$ containing $U$. Since the winding number of each JSJ torus $T_{i}$ is nonzero with respect to $T_{0}$, we have $S \cap T_{i} \neq \emptyset$ for each $i$, and it follows that $f^{-1}\left(X_{0}^{\prime}\right) \cap U=U$.

Claim 3. $U=E\left(p_{1}, q_{1} ; \ldots ; p_{n}, q_{n}\right)$.

Proof. If $U=C\left(p_{1}, q_{1} ; \ldots ; p_{n}, q_{n}\right)$. Let $Y$ be the JSJ piece of $E(k)-U$ adjacent to $T_{n}$. By the definition of $U, Y$ must be a hyperbolic piece, so $f \mid Y$ must be a homeomorphism. Since $f\left(T_{n}\right) \subset T_{0}^{\prime}$, we must have $f(Y) \subset X^{\prime}$ and it implies that $X^{\prime}$ is a hyperbolic piece. Since $f: X \rightarrow X^{\prime}$ is homeomorphism by our assumption, it follows that $X$ is a hyperbolic piece. Therefore $f$ send two different hyperbolic JSJ pieces of $E(k)$ to one hyperbolic JSJ piece of $E\left(k^{\prime}\right)$, it contradicts that $f \mid$ on the hyperbolic part is a homeomorphism.

Now we have a proper map $f: E\left(p_{1}, q_{1} ; \ldots ; p_{n}, q_{n}\right) \rightarrow E\left(p^{\prime}, q^{\prime}\right)$ which is a homeomorphism on the boundary. By Lemma 2.7, $f \mid T_{1}$ is not $\pi_{1}$-injective, which contradicts to the assumption we made before.

This finishes the proof of Lemma 3.2 . 
Lemma 3.3. $f \mid T_{1}$ is not $\pi_{1}$-injective.

Proof. By Lemma [3.2, some $f \mid T_{i}$ is not $\pi_{1}$-injective for $T_{i}$ in $U$. We may assume that $f \mid$ is $\pi_{1}$-injective on $T_{i}$ for $i<k$ and that $f \mid$ is not $\pi_{1}$-injective on $T_{k}$. We have $f\left(C\left(p_{1}, q_{1} ; \ldots ; p_{k}, q_{k}\right)\right) \subset X_{0}^{\prime}$. Since $f \mid T_{k}$ is not $\pi_{1}$-injective, there is a simple loop $\alpha \in T_{i}$ in the kernel of $f_{*}$. Therefore we get a map $f \mid: C\left(p_{1}, q_{1} ; \ldots ; p_{k}, q_{k} ; \alpha\right) \rightarrow X_{0}^{\prime}$ such that $f \mid T_{0}$ is a homeomorphism. A homological argument shows that $X_{0}^{\prime}=E(p, q)$. By Lemma $2.7 f \mid T_{1}$ is not $\pi_{1}$-injective.

Proof of Theorem 1.1. Let $V=M\left(\Gamma_{0}\right), V^{\prime}=f(V)$. Then $f \mid: V \rightarrow V^{\prime}$ is a homeomorphism. Denote the knot complement separated by $T_{i}$ in $E(k)$ by $E\left(k_{i}\right), i=0,1$ and $W=E(k) \backslash E\left(k_{0}\right)$. Then we have $E\left(k_{0}\right)=C\left(p_{1}, q_{1}\right) \cup_{T_{1}}$ $E\left(k_{1}\right)$ and there is a proper degree one map

$$
f: E(k)=W \cup_{T_{0}} C\left(p_{1}, q_{1}\right) \cup_{T_{1}} E\left(k_{1}\right) \rightarrow E\left(k^{\prime}\right)
$$

such that $f\left(C\left(p_{1}, q_{1}\right)\right) \subset X_{0}^{\prime}, f \mid: T_{0} \rightarrow T_{0}^{\prime}$ is a homeomorphism, and a simple closed curve $\alpha \subset T_{1}$ lies in the kernel of $f \mid T_{1}$. Then the proper degree one map $f: E(k) \rightarrow E\left(k^{\prime}\right)$ induces a factorization

$$
E(k) \longrightarrow W \cup_{T_{0}} C\left(p_{1}, q_{1} ; \alpha\right) \cup_{\alpha^{*}} E\left(k_{1}, \alpha\right) \stackrel{\hat{f}}{\longrightarrow} E\left(k^{\prime}\right) .
$$

Here $C\left(p_{1}, q_{1} ; \alpha\right)$ and $E\left(k_{1}, \alpha\right)$ are 3 -manifolds obtained by Dehn filling along $\alpha \subset T_{1}$ on $C\left(p_{1}, q_{1}\right)$ and $E\left(k_{1}\right)$ respectively and $C\left(p_{1}, q_{1} ; \alpha\right) \cup_{\alpha^{*}} E\left(k_{1}, \alpha\right)$ is obtained by identifying the core $\alpha^{*}$ of filling solid tori in $C\left(p_{1}, q_{1} ; \alpha\right)$ and $E\left(k_{1}, \alpha\right)$.

Since $E\left(k_{1}, \alpha\right)$ is a closed 3 -manifold, it makes no contribution to the degree of the proper degree one map $f$ and we have

$$
\hat{f} \mid: W \cup_{T_{0}} C\left(p_{1}, q_{1} ; \alpha\right) \rightarrow E\left(k^{\prime}\right)
$$

is a proper degree one map. Since

$$
\left\|E\left(k^{\prime}\right)\right\|=\|E(k)\| \geq\left\|W \cup_{T_{0}} C\left(p_{1}, q_{1}\right)\right\| \geq\left\|W \cup_{T_{0}} C\left(p_{1}, q_{1} ; \alpha\right)\right\| \geq\left\|E\left(k^{\prime}\right)\right\|,
$$

we have $\|E(k)\|=\left\|W \cup_{T_{0}} C\left(p_{1}, q_{1}\right)\right\|$ and therefore $E\left(k_{1}\right)$ is a graph manifold, it follows that

$$
C\left(p_{1}, q_{1}\right) \cup_{T_{1}} E\left(k_{1}\right)=C\left(p_{1}, q_{1}\right) \cup_{T_{1}} E\left(p_{2}, q_{2} ; \ldots ; p_{n}, q_{n}\right)
$$


where $C\left(p_{1}, q_{1}\right) \cup_{T_{1}} E\left(p_{2}, q_{2} ; \ldots ; p_{n}, q_{n}\right)=E\left(p_{1}, q_{1} ; \ldots ; p_{n}, q_{n}\right)$

Moreover since $\hat{f}\left(C\left(p_{1}, q_{1} ; \alpha\right)\right) \subset X_{0}^{\prime}, f \mid: T_{0} \rightarrow T_{0}^{\prime}$ is a homeomorphism, it follows that $X_{0}^{\prime}=E\left(p^{\prime}, q^{\prime}\right)$ and

$$
\hat{f} \mid: C\left(p_{1}, q_{1} ; \alpha\right) \rightarrow E\left(p^{\prime}, q^{\prime}\right)
$$

is homotopic to a homeomorphism. Finally we have

$$
f: W \cup_{T_{0}} C\left(p_{1}, q_{1}\right) \cup_{T_{1}} E\left(p_{2}, q_{2} ; \ldots ; p_{n}, q_{n}\right) \rightarrow E\left(k^{\prime}\right)=W^{\prime} \cup_{T_{0}^{\prime}} E\left(p^{\prime}, q^{\prime}\right) .
$$

Let $S^{\prime}$ be a Seifert surface of $E\left(p^{\prime}, q^{\prime}\right)$, then up to a homotopy relative to $T_{0}$, we may assume that $f^{-1}\left(S^{\prime}\right)$ is incompressible, and moreover there is only one component of $f^{-1}\left(S^{\prime}\right)$, denoted by $S$, with $\partial S$ a circle. Let $X$ be a JSJ piece of $E(k)$ adjacent to $X_{0}$ along $T_{0}$, and let $X^{\prime}$ be a JSJ piece of $E\left(k^{\prime}\right)$ adjacent to $X_{0}^{\prime}$ along $T_{0}^{\prime}$. By our choice of $T_{0}, f \mid X$ is a homeomorphism. Since $X$ has at least two boundary components while $X_{0}^{\prime}$ has only one boundary component, we must have $f(X) \subset X^{\prime}$ and therefore $f^{-1}\left(S^{\prime}\right) \cap$ int $X=\emptyset$. Since $T_{0}$ is separating and $S$ is connected, we must have $S \subset E\left(p_{1}, q_{1} ; p_{2}, q_{2} ; \ldots ; p_{n}, q_{n}\right)$ and therefore it is a Seifert surface of $E\left(p_{1}, q_{1} ; p_{2}, q_{2} ; \ldots ; p_{n}, q_{n}\right)$ which intersects $T_{1}$ in parallel copies of $\lambda_{1}$. It follows that $\alpha=\lambda_{1}$. Now we rewrite (1) as

$$
E(k) \longrightarrow V \cup_{T_{0}} C\left(p_{1}, q_{1} ; \lambda_{1}\right) \cup_{\lambda_{1}^{*}} E\left(k_{1}, \lambda_{1}\right) \stackrel{\hat{f}}{\longrightarrow} W^{\prime} \cup_{T_{0}^{\prime}} E\left(p^{\prime}, q^{\prime}\right) .
$$

Note that the core $\lambda_{1}^{*}$ of the filling solid torus is a retractor of $E\left(k_{1}, \lambda_{1}\right)$, and $\hat{f} \mid: C\left(p_{1}, q_{1} ; \lambda_{1}\right) \rightarrow E\left(p^{\prime}, q^{\prime}\right)$ is homotopic to a homeomorphism by [Ro]. Now we have a further factorization

$$
\begin{aligned}
E(k) & \rightarrow W \cup_{T_{0}} C\left(p_{1}, q_{1} ; \lambda_{1}\right) \cup_{\lambda_{1}^{*}} E\left(k_{1}, \lambda_{1}\right) \\
& \rightarrow W \cup_{T_{0}} C\left(p_{1}, q_{1} ; \lambda_{1}\right)=W \cup_{T_{0}} E\left(p^{\prime}, q^{\prime}\right) \rightarrow W^{\prime} \cup_{T_{0}^{\prime}} E\left(p^{\prime}, q^{\prime}\right) .
\end{aligned}
$$

Hence $f$ factors through the de-satellization:

$$
E(k) \rightarrow W \cup_{T_{0}} E\left(p^{\prime}, q^{\prime}\right) \rightarrow E\left(k^{\prime}\right) .
$$

Clearly $W \cup_{T_{0}} E\left(p^{\prime}, q^{\prime}\right)=E\left(k^{\prime \prime}\right)$ for some knot $k^{\prime \prime}$ in $S^{3}$. Moreover any companion of $k^{\prime \prime}$ is prime, $k^{\prime \prime}$ and $k^{\prime}$ have the same simplicial volume. So we can repeat the above process to degree one map $E\left(k^{\prime \prime}\right) \rightarrow E\left(k^{\prime}\right)$. Since any knot admits at most finitely many de-satellization, we finish the proof of Theorem 1.1. 


\section{New construction}

Example 4.1. We construct a degree one map from a graph knot (i.e., the complement of the knot is a graph manifolds) to a torus knot which is not a de-satellization.

Below $c_{i}$ and $P_{n}$ are given in Notation 1.5, We use $\bar{T}(3,2)$ to denote the mirror image of $T(3,2)$ and $\bar{E}(3,2)$ to donote the exterior of $\bar{T}(3,2)$.

Lemma 4.2 (Schubert). The JSJ-decomposition pieces of $E\left(k_{1} \# \ldots \# k_{n}\right)$ are $E\left(k_{1}\right), \ldots, E\left(k_{n}\right)$ and $P_{n} \times S^{1}$, moreover $E\left(k_{1} \# \ldots \# k_{n}\right)$ is obtained by identifying $\partial E\left(k_{i}\right)$ and $c_{i} \times S^{1}$ such that the meridian $m_{i}$ of $E\left(k_{i}\right)$ is identified with $x_{i} \times S^{1}$, where $x_{i}$ is a point in $c_{i}, i=1, \ldots, n$.

To construct our example, we need first to orient knot exteriors and their meridians and Seifert fibers and to take a careful look at Lemma 4.2.

The orientation of each knot exterior below is induced from the 3 -sphere with fixed orientation; the torus boundary of each knot exterior has induced orientation; on each torus boundary, the meridian and the Seifert fiber are oriented so that their product give the orientation of the torus.

Suppose the meridian and the Seifert fiber of $E(3,2)$ have been oriented.

Lemma 4.3. (i) The meridian and the Seifert fiber of $E(3 p, 2)$ can be oriented so that there is a proper map

$$
\pi_{p}: E(3 p, 2) \rightarrow E(3,2)
$$

of degree $p$ for any odd $p$ which sends the Seifert fiber of $E(3 p, 2)$ to the $p$ times of Seifert fiber of $E(3,2)$ and send the meridian to the meridian.

(ii) The meridian and the Seifert fiber of $\bar{E}(3,2)$ can be oriented so that there is a proper degree -1 map

$$
\bar{\pi}: \bar{E}(3,2) \rightarrow E(3,2)
$$

which send the meridian to the meridian and reverses the direction of the Seifert fiber.

Proof. (i) Let $A$ be a cyclic group of order $p$ acts freely on along the regular Seifert fiber on $E(3 p, 2)$ which induces the identity on the base space. One can verify directly that the quotient $E(3 p, 2) / A=E(3,2)$ for odd $p$. Moreover 
if we lift the orientations of the meridian and the Seifert fiber of $E(3 p, 2)$ to those of $E(3 p, 2)$, then the quotient map $\pi_{p}: E(3 p, 2) \rightarrow E(3,2)$ meets all the conditions.

(ii) By the definition there is a proper degree -1 map

$$
r: \bar{E}(3,2) \rightarrow E(3,2)
$$

induced by the mirror reflection. Now orient the meridian and the Seifert fiber of $\bar{E}(3,2)$ so that $r$ reverses the direction of meridian and preserves the oriented Seifert fiber. Since the trefoil knot is strongly invertible, there is orientation preserving involution $\tau$ which reverses both the directions of the Seifert fiber and the meridian on $\partial E(3,2)$. Then the composition $\bar{\pi}=\tau \circ r$ meets all the conditions.

In the next lemma, $P_{n}$ 's are oriented and $\partial P_{n}$ 's have induced orientations. The proof of the lemma is very direct.

Lemma 4.4. Let $d_{1}, \ldots, d_{n}$ be integers such that $\sum d_{i}=1$. There is a proper degree one map $h\left(d_{1}, \ldots, d_{n}\right):\left(P_{n}, c_{0}, \cup_{i=1}^{n} c_{i}\right) \rightarrow\left(P_{1}, c_{0}, c_{1}\right)$ such that the restriction $h \mid: c_{0} \rightarrow c_{0}$ is of degree 1 and $h \mid: c_{i} \rightarrow c_{1}$ is of degree $d_{i}$.

Now we are going to construct a degree one map

$$
f: E(T(9,2) \# \bar{T}(3,2) \# \bar{T}(3,2)) \rightarrow E(3,2)
$$

which we call "folding". To define the map, we need to present the domain and the target as follows:

$$
f:\left(P_{3} \times S^{1}\right) \cup_{\phi_{i}} \sqcup_{i=1}^{3} E_{i} \rightarrow\left(P_{1} \times S^{1}\right) \cup_{\phi} E(3,2)
$$

where $E_{1}=E(9,2), E_{2}=\bar{E}(3,2), E_{3}=\bar{E}(3,2)$, and take a careful look at $\phi_{i}$ and $\phi$.

First all the meridians and the Seifert fibers of $E_{i}, i=1,2,3$, are oriented as in Lemma 4.3 and all $c_{i}$ are oriented as in Lemma 4.4, and $S^{1}$ is also oriented.

Now each $\phi_{i}$ exactly sends the meridian of $E_{i}$ to $x_{i} \times S^{1}$. Moreover the product structure of $P_{3} \times S^{1}$ can be chosen so that $\phi_{i}$ sends the Seifert fiber of $E_{i}$ to $c_{i} \times y$, which is possible since the Seifert fiber and the meridian of $E_{i}$ meets transversally in one point. The product structure of $P_{1} \times S^{1}$ is also chosen so that $\phi$ has similar property. 
Now our map $f$ is obtained by gluing the following proper maps:

(1) $h(3,-1,-1) \times i d: P_{3} \times S^{1} \rightarrow P_{1} \times S^{1}$, where $h(3,-1,-1)$ is defined in Lemma 4.4;

(2) $\pi_{3}: E_{1} \rightarrow E(3,2)$, where $\pi_{3}$ is given by Lemma 4.3 (i);

(3) $\bar{\pi}: E_{i} \rightarrow E(3,2)$, where $\bar{\pi}$ is given by Lemma 4.3 (ii), $i=2,3$.

Clearly $f$ is a proper map of degree one.

Finally we show that the map $f$ is not a de-satellization. Otherwise there would be an essential embedded torus $T$ such that there is a non-trivial simple closed curve $c$ which stays in the kernel of $f_{*}$. Since all $E_{i}$ involved are small knot exteriors, $T \subset E(k)$ must be a vertical torus in $P_{3} \times S^{1}$, which separates $P_{3} \times S^{1}$ into two copies of $P_{2} \times S^{1}$. We may that suppose $c_{1}$ and $c_{2}$ are in the same $P_{2} \times S^{1}$. Note that $f$ send $\left(S^{1}, c_{1}, c_{2}\right)$ of $P_{2} \times S^{1}$ to $\left(S^{1}, 3 c_{1}\right.$, $\left.-c_{1}\right)$ of $P_{1} \times S^{1}$, and $c_{1}$ and $S^{1}$ form a basis for $\pi_{1}\left(P_{1} \times S^{1}\right)$, one can verify directly that there is no non-trivial simple closed curve on $T$ which stays in the kernel of $f \mid: T \rightarrow P_{1} \times S^{1}$. Since $P_{1} \times S^{1}$ is $\pi_{1}$-injective in $E(3,2)$, so there is no non-trivial simple closed curve on $T$ which stays in the kernel of $f \mid: T \rightarrow E(3,2)$, and we reach a contradiction. The verification of the cases that other $c_{i}$ and $c_{j}$ are in the same $P_{2} \times S^{1}$ is similar.

\section{References}

[BNW] M. Boileau, Y. Ni and S. C. Wang, Pseudo-Anosov extensions and degree one maps between hyperbolic surface bundles, Math. Z. 256 No. 4, 2007, 913-923.

[BW1] M. Boileau and S.C. Wang, Non-zero degree maps and surface bundles over $S^{1}$, J. Differential Geom. 43, 1996, 789-806.

[BW2] M. Boileau and S.C. Wang, Degree one maps between small hyperbolic 3manifolds, Proc. Amer. Math. Soc. 135 2007, 4023-4029.

[Go] C. Gordon, Dehn surgery and satellite knots, Trans. Amer. Math. Soc. 275, 1983, 687-708.

[GL] C. Gordon and J. Luecke, Knots are determined by their complements, J. Amer. Math. Soc. (2) 2, 1989, 371-415.

[He] J. Hempel, 3-manifolds, Princeton University Press, 1976.

[Ja] W.H. Jaco, Lectures on three-manifold topology, CBMS Regional Conference Series in Mathematics, 43. American Mathematical Society, Providence, R.I., 1980 .

[Ka] A. Kawauchi, Almost identical imitations of (3,1)-dimensional manifold pairs, Osaka J. Math. 26 1989, no. 4, 743-758. 
[ORS] T. Ohtsuki, R. Riley and M. Sakuma, Epimorphisms between 2-bridge link groups, Manuscript, June 2006. To be published in a volume of GT Monograph in meomory of Heiner Zieschang.

[R] D. Rolfsen, Knots and Links, Mathematics Lecture Series, No. 7. Publish or Perish, Inc., Berkeley, Calif., 1976.

[Ro] Y. Rong, Maps between Seifert fibered spaces of infinite $\pi_{1}$. Pacific J. Math. 160 (1993), no. 1, 143-154.

[Ru] D. Ruberman, Seifert surfaces of knots in $S^{4}$, Pacific J. Math. 145, 1990, 97-116.

[Sc] H. Schubert, Über eine numerische Knoteninvariante. (German) Math. Z. 61 (1954), 245-288.

[So] T. Soma, A rigidity theorem for Haken manifolds. Math. Proc. Cambridge Philos. Soc. 118 (1995), no. 1, 141-160.

[Wal] F. Waldhausen, On irreducible 3-manifolds which are sufficiently large, Ann. of Math. (2) 87 (1968), 56-88.

[W] S. C. Wang, Non-zero degree maps between 3-manifolds, Proceedings of the International Congress of Mathematicians, Vol. II (Beijing, 2002), 457-468, Higher Ed. Press, Beijing, 2002. 